\title{
Review of: "Open Science"
}

\author{
Ameena Payne
}

Potential competing interests: The author(s) declared that no potential competing interests exist.

My only suggestion would be to provide a link for more information such as the UN's Open Science Movement:

http://www.unesco.org/new/en/communication-and-information/portals-and-platforms/goap/open-science-movement/ 